The Journal of Animal \& Plant Sciences, 30(5): 2020, Page: 1283-1291

ISSN (print): 1018-7081; ISSN (online): 2309-8694

\title{
A COMPARISON OF GENE CO-NETWORKS REVEALED THE MOLECULAR MECHANISMS OF FLORAL TRANSITION AND INFLORESCENCE DEVELOPMENT IN BARLEY (HORDEUM VULGARE L.)
}

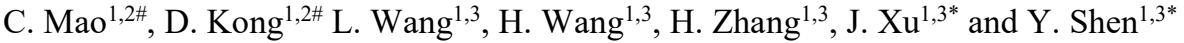 \\ ${ }^{1}$ Key Laboratory of Adaptation and Evolution of Plateau Biota, Qinghai Provincial Key Laboratory of Crop Molecular \\ Breeding, Northwest Institute of Plateau Biology, Chinese Academy of Sciences, Xining 810008, China \\ ${ }^{2}$ University of Chinese Academy of Sciences, Beijing 100049, China \\ ${ }^{3}$ Innovation Academy for Seed Design, CAS, Xining 810008, China \\ *Corresponding Author's E-mail: xjq1088@126.com; shenyuhu@nwipb.cas.cn
}

\begin{abstract}
Inflorescence development and flower organ development are important events in plant development, directly affecting sexual reproduction and plant yield. The molecular mechanism of the inflorescence development in barley (Hordeum vulgare L.) has rarely been studied. In order to mine the genes involved in the development of barley inflorescence, published RNA-seq data were analyzed by weighted gene co-expression network analysis (WGCNA), and six modules were identified. Further analysis showed that three modules (green, light green and purple) were significantly associated with the stage during floral development. The module-specific genes were enriched in physiological processes such as transporter activity, metal binding and oxidoreductase activity. In barley, the genes encoding the $M A D S$-box transcription factor family protein, REDUCED VERNALIZATION RESPONSE1 (VRN1) and ASYMMETRIC LEAVES1 were identified as hub genes in the corresponding modules, and were reported previously to be connected with vegetative apices, leaf growth and auxin signaling. In addition, we also found candidate genes encoding AP2/B3-like transcriptional factor family protein, proline-rich family protein, growth-regulating factor 2 (GRF2), armadillo-repeat containing protein (ARP), xyloglucan endotransglucosylase/hydrolase 9 (XTH9) and G2484-1. This work discovered candidate genes that highly related to the inflorescence development in barley using the constructed genetic network relationship, and provided new ideas and datasets for studying the molecular mechanism of barley inflorescence development.
\end{abstract}

Key word: Barley, Co-expression network analysis, Inflorescence development, Transcriptomics.

https://doi.org/10.36899/JAPS.2020.5.0146

Published online June 25, 2020

\section{INTRODUCTION}

In flowering plants, phase transition from vegetative to reproductive growth is a critical event in the plant life cycle. The time of developmental events before flowering affects the number of grains per plant, which is directly related to yield traits (Fischer, 1985; Sebastián and Miralles, 2008). Some studies on wheat (Triticum L.) and barley (Hordeum vulgare L.) have shown that the duration of preanthesis in different phenological periods is genetically controlled and they have a variety of sensitivity of vernalization and photoperiod (Slafer and Rawson, 1994; González et al., 2002; Borràs-Gelonch et al., 2012). The Ppd-H1 gene is located on the short arm of the $2 \mathrm{H}$ chromosome of barley. This gene belongs to the PRR (Pseudo-response regulator) gene family and contains the pseudo-receiver and CCT domains of signal transduction. Due to point mutations in the CCT domain, the encoded glycine (Gly) becomes tryptophan (Trp), causing long-day insensitivity. $\mathrm{Ppd}-\mathrm{Hl}$ is a gene that responds to long-day sunlight in barley and promotes heading under long days, while $p p d-H 1$ is relatively late maturing. Under long-day conditions, plants with $\mathrm{Ppd}-\mathrm{HI}$ are eared about 20 days earlier than those with $p p d-H 1$ (Turner et al., 2005; Kitagawa et al., 2012; Seki et al., 2013). In barley, $P p d-H 1$ is often found in winter products, and $p p d-H 1$ is often found in spring varieties (Laurie et al., 1995). Wild barley varieties are extremely sensitive to long-day sunlight and mature early under long days (Karsai et al., 2004). The sensitivity of springgrown cultivars to sunlight depends on their origin and area of cultivation.

Weighted gene co-expression network analysis (WGCNA) is a co-expression network analysis method that transforms the connection relationship from "yes/no" to connection weights, using a biologically significant criterion-scale-free topology criterion (Langfelder and Horvath, 2008). The relationships among genes are changed from being general to having varying degrees of connectivity. The highly related genes are divided into one module by calculation, and different modules are associated with similar corresponding phenotypes. Studying the genes in the module by reversing the desired phenotype is actually a new approach to reverse genetics. A large number of published RNA-seq data can be used 
to reveal potential phenotype-related genes, and WGCNA has been shown to be effective in the discovery of previously unscreened specific expression genes. (Bao et al., 2017).

WGCNA has achieved reliable results under a variety of biological environments (Ping et al., 2015; Giulietti et al., 2017; Lin et al., 2018). In agricultural research, it can be used to mine genes with higher correlations with specific phenotypes, i.e., inflorescence development (Feng et al., 2017), fruit ripening (Ghan et al., 2017) and response to stress (Wang et al., 2014). The use of the WGCNA method to study barley-related phenotypes has only been used to a limited extent thus far, including the studies of starch biosynthesis during barley development and response to powdery mildew (Tang et al., 2017; Yuan et al., 2018). In this study, we used WGCNA to reanalyze the published RNA-seq data for barley inflorescence (Digel et al., 2015), which can be used to confirm previous conclusions. It is also possible to discover candidate genes highly related to inflorescence development in the constructed genetic network relationship.

\section{MATERIALS AND METHODS}

Data: The research data PRJEB8748 is derived from the Sequence Read Archive (SRA) database of NCBI, including 29 samples collected from main shoot apices (MSAs). Details of the samples can be found in Supplementary Table 1. The Reads Per Kilobase per Million mapped reads (RPKM) values of the genes were based on the results of Supplemental Data set 3 in Digel et al (Digel et al., 2015).

Methods: We used the $\mathrm{R}$ software (v. 3. 4. 1) and WGCNA (v. 1. 49) software package to construct a weighted gene expression network and to divide the modules (Langfelder and Horvath, 2008). First, the average value of RPKM in the three replicates of each gene was calculated, and the top 3,000 genes were used for further analysis. After clustering the samples with the average method, we set the cut height to 3,000 to remove outliers. As shown in Supplementary Fig. 1, 4 outliers were detected. Then we created a sample dendrogram and used the corresponding trait heatmap to verify that the remaining 25 samples exactly matched the phenotype (Supplementary Fig. 2).

A proper adjacency matrix weight parameter $\beta$ value was determined by correlation coefficient and gene connectivity. Using Supplementary Fig. 3, we chose the leading matrix weight parameter $\beta=22$. Then we constructed a WGCNA network using the automatic network constructor block wise modules. The branches of each cluster tree form a gene module, and each branch in the cluster tree branch represents a gene. Using the dynamic shearing method, genes with higher similarity
(0.8) were defined as a module and were assigned corresponding colors. Screening for genomes (modules) with the same expression pattern based on their correlation with phenotype. Then we made a correlation analysis between the module genes and traits, and the modules with correlation coefficients $>0.45$ were selected to analyze their connection with floral development (Zhang et al., 2018). Furthermore, GO (Gene ontology) annotation for each gene in these modules was applied from Supplemental Data Set 2 of Digel et al. (2015). GO enrichment analysis was performed using the OmicShare tools, a free online platform for data analysis (www.omicshare.com/tools).

The hub genes refer to genes with high connectivity in the module, which are more representative than other genes in the module and can be used for differential expression analysis between different materials (treatment). To further explore the interaction of genes within the module, we used intra-modular linkage (kIN) to look for key genes from the module and selected those genes with connectivity $>0.2$ to map the gene network.

\section{RESULTS}

Gene network constructed by WGCNA: WGCNA divided all genes into 28 modules based on the optimal $\beta$ of 22. As shown in Fig. 1, after using the dynamic shearing method only six modules were retained. The number of genes divided by each module ranges from 202 to 1,464 (Fig. 2A). The matrix representing all Module-trait relationships (MTRs) is different. These modules include a large number of positively or negatively related genes and having a high or low expression levels at different stages of development of the inflorescence.

Major modules correlated with different stages of floral development: Due to S42-IL107 (S1) is an introgression line with a photoperiod-responsive dominant Ppd-H1 allele, the genetic background of S42IL107 and Scarlett (S2) is similar. We identified three modules significantly associated with $\mathrm{S} 1$. The purple module $(r=0.77, p=8 \mathrm{e}-06)$ was highly correlated with the W3.5 stage under LD (long-day) conditions, and the green module under LD conditions showed high correlation $(r=0.48, p=0.01)$ with the W1 stage (Fig. $2 \mathrm{~A})$. The light green module was highly correlated with the SD (short-day) conditions at the W3.5 $(r=0.46, p=$ $0.02)$ stage and the W1 stage $(r=0.61, p=0.001)$, respectively (Fig. 2A).

Hub gene selection for S1 co-expression networks: As the heatmaps displayed (Fig. 3A), green-module-specific genes were overrepresented in the W0.5 and W1 stages in $\mathrm{S} 1$. Eigen-gene expression profiles for the green module are shown in Fig. 3B and the correlation network of green 
module are shown in Fig. 3C. For green module-specific genes, the GO term is rich in sequence-specific DNA binding, transcription factor activity and nucleic acidbinding transcription factor activity. (Fig. 4). Among the hub genes in this module, seven genes encoding transcription factor (TF), including three MADS-box TFs (Hv.15491, Hv.19680,Hv.110), two AP2/B3-like TFs (Hv.12609, MLOC_54060.1), one bZIP $\mathrm{TF}\left(M L O C_{-} 14596.3\right)$, one NAC TF $(H v .35231)$ and one BEL1-like homeodomain 11 (BLH11, Hv.34830). Genes encoding growth-regulating factor 2 (GRF2, Hv.15756), phytochrome-associated protein 1 (PAP1, Hv.31348), LIGHT SENSITIVE HYPOCOTYS 3 (LSH3, Hv.17107), LSH6 (Hv.34251) were also found. Three genes, VEGETATIVE TO REPRODUCTIVE TRANSITION2 (VRT2; Hv.15491), BARLEY MADS BOX10 (BM10; Hv.19680), and a homolog of REDUCED VERNALIZATION RESPONSE 1 (VRN1; Hv.12609) were in the center of green-module network (Fig. 3C).

Light green module-specific gene heatmaps (Fig. 3D) were overrepresented in the W2 and W3.5 stages. Eigen-gene expression profiles for the light green module are shown in Fig. $3 \mathrm{E}$ and Fig. $3 \mathrm{~F}$ reveals the correlation network of this module. GO enrichment demonstrated that genes in the light green module were significantly enriched in GO terms with metal binding, such as zinc ion binding, transition metal ion binding, metal ion binding, ion binding and cation binding (Fig. 4). Twenty-four of the 30 hub genes in the light green module were annotated, including one $A P 2 / B 3$-like $T F$ (Hv.11103), one BTB/POZ/NATH-domains containing protein (Hv.11353), two $\mathrm{C} 2 \mathrm{H} 2$-like zinc finger proteins (Hv.8749, Hv.13792), one F-box/RNI-like/FBD-like domains-containing protein ( $H v .19756)$, one CCCH-type zinc finger family protein $(H v .2670)$ and one
RING/FYVE/PHD zinc finger superfamily protein (MLOC_60146.1). More importantly, we found two genes directly associated with flowering, $H v .17103$ encodes armadillo-repeat containing protein (ARM), which is involved in leaf and flower development; $H v .4496$ encodes a proline-rich family of proteins, a nuclear targeting protein that is involved in the regulation of inflorescence development and affects flowering time, independent of FLC (FLOWERING LOCUS C). As shown in Fig. 3F Hv.4496 was in the center of the light green module network (Fig. 3F).

For S1, heatmaps (Fig. 3G) showed that purple module-specific genes were overrepresented in the W2 and W3.5 stages. The characteristic gene expression profile of the purple module is shown in Figure. 3 H. The GO terms of genes in this module were enriched in oxidoreductase activity, monovalent inorganic cation transmembrane transporter activity, and inorganic cation transmembrane transporter activity (Fig. 4). In total, three genes encode TFs: one MYB-like HTH transcriptional regulator family protein $(H v .12064)$, one zinc finger (CCCH-type) family protein (Hv.9817), and one zinc finger (C2H2-type) (MLOC_60374.1). In addition, we also found genes encoding xyloglucan endotransglucosylase/hydrolase 9 (XTH9, Hv.12170), gamma tonoplast intrinsic protein (MLOC_73301.3), GYF domain-containing protein $(H v .1693)$ and polygalacturonase inhibiting protein 1 (Hv.1123). $H v .12064$ and $H v .1878$ were in the center of the purple correlation network. Hv.12064 encodes a MYB domain protein that is involved in leaf growth and auxin signaling (Digel et al., 2015), while Hv.1878 encodes G2484-1 protein, indicating this protein maybe provide new clues for understanding the mechanism of floral development (Fig. 3I).

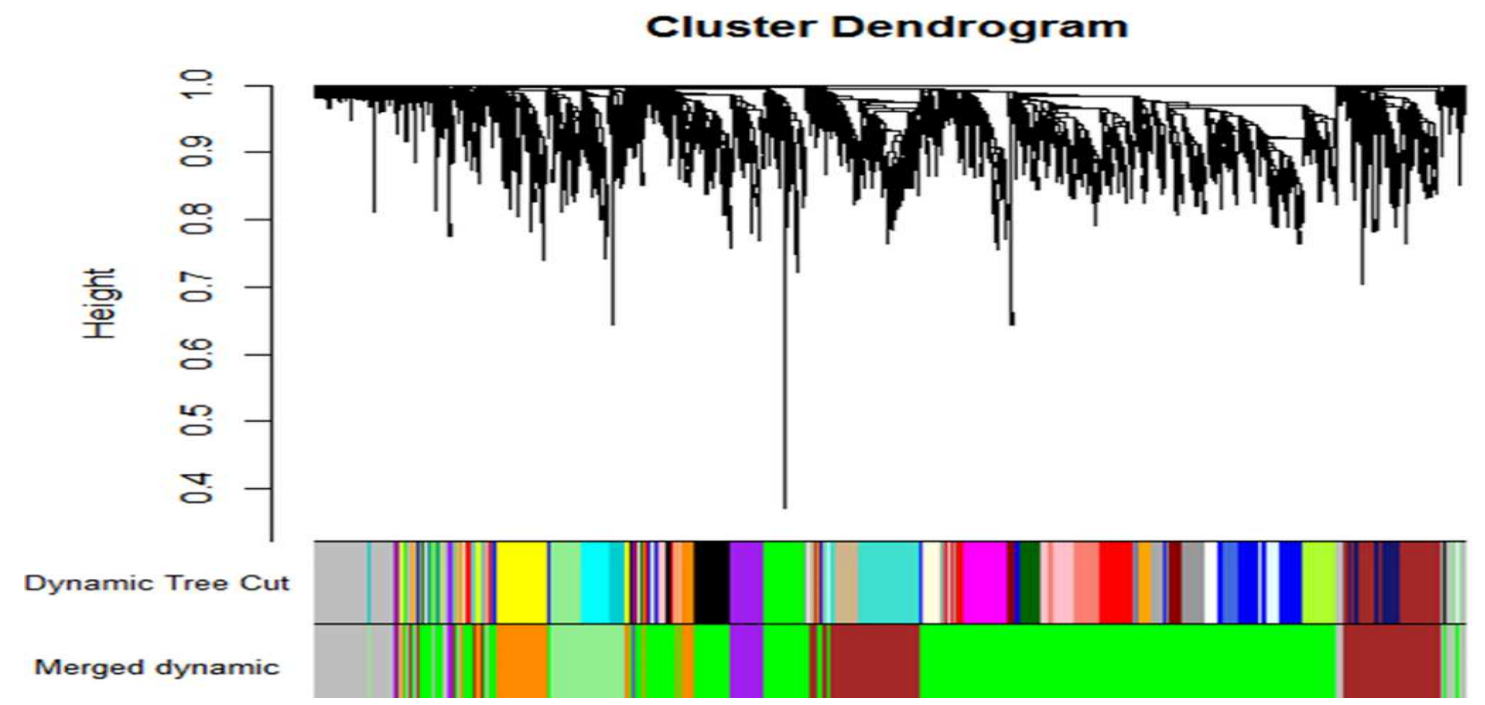

Fig. 1. Hierarchical cluster tree showing co-expression modules identified by WGCNA. Each leaf in the tree represents one gene. The major tree branches constitute 6 modules, labeled with different colors. 
A

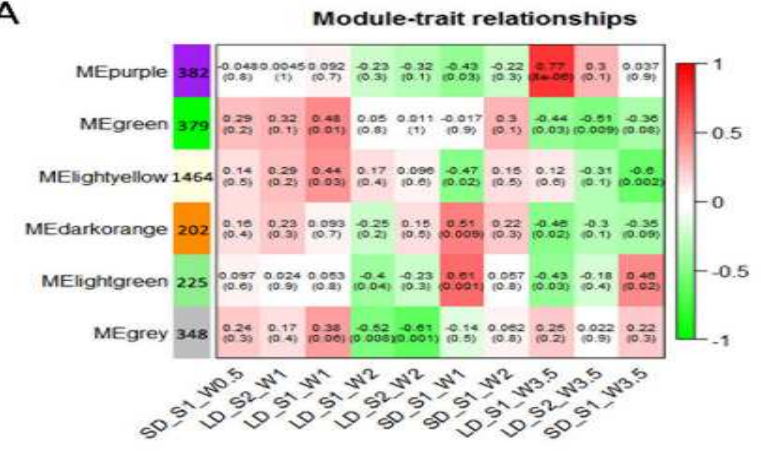

C

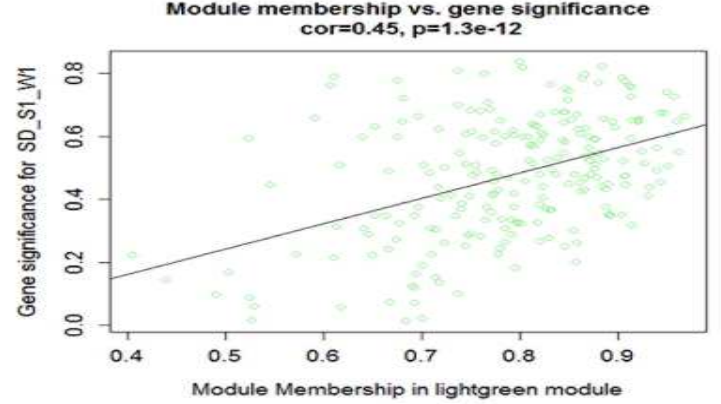

B

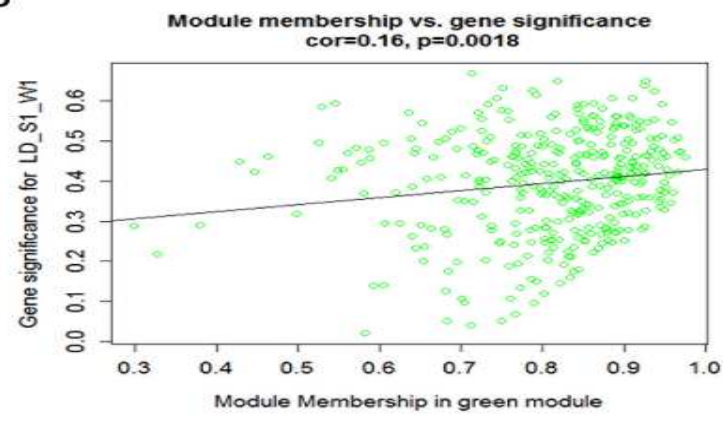

D

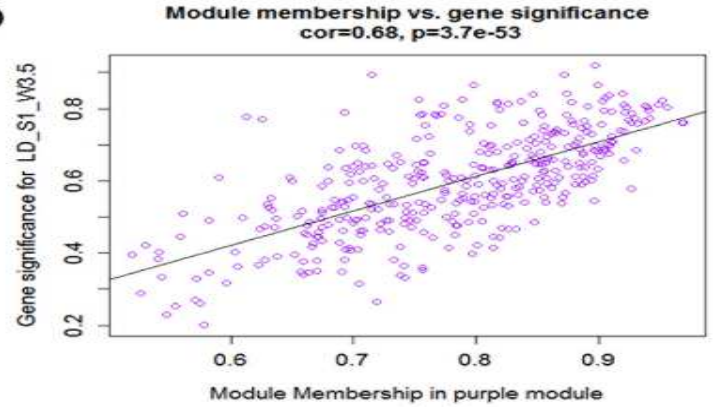

Fig. 2. Module-Trait Relationships (MTRs) and gene significance in selected modules. A) Matrix of module-trait relationships. Each row corresponds to a module. The number of genes in each module is indicated on the left. Each column corresponds to a specific treatment. MTRs are colored based on their correlation. A high degree of correlation is indicated by dark red and dark green. B-D) Scatterplots of gene significance versus the selected modules (green, light green and purple).
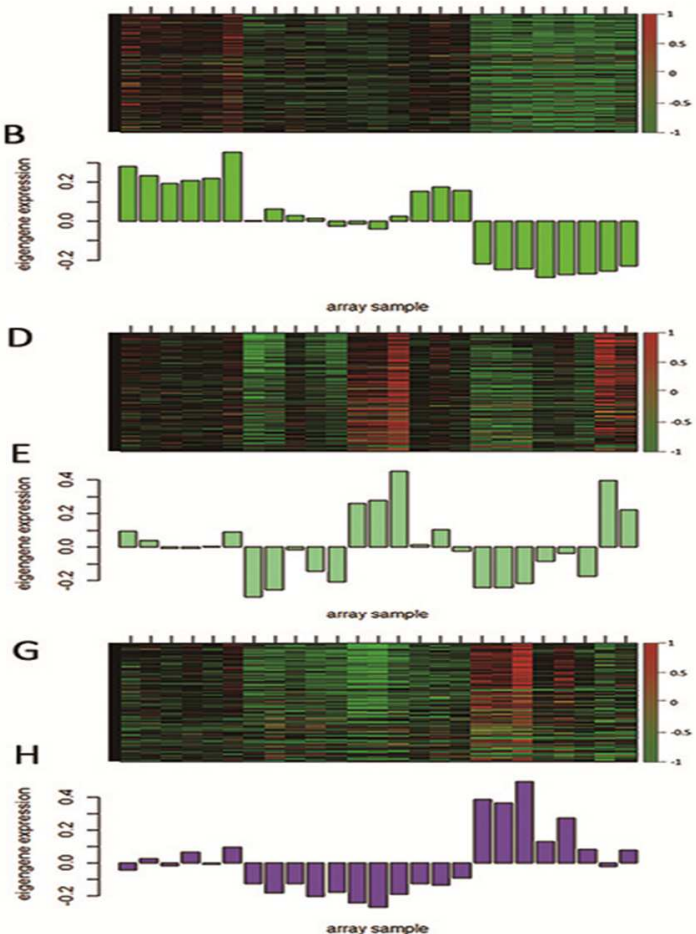

C
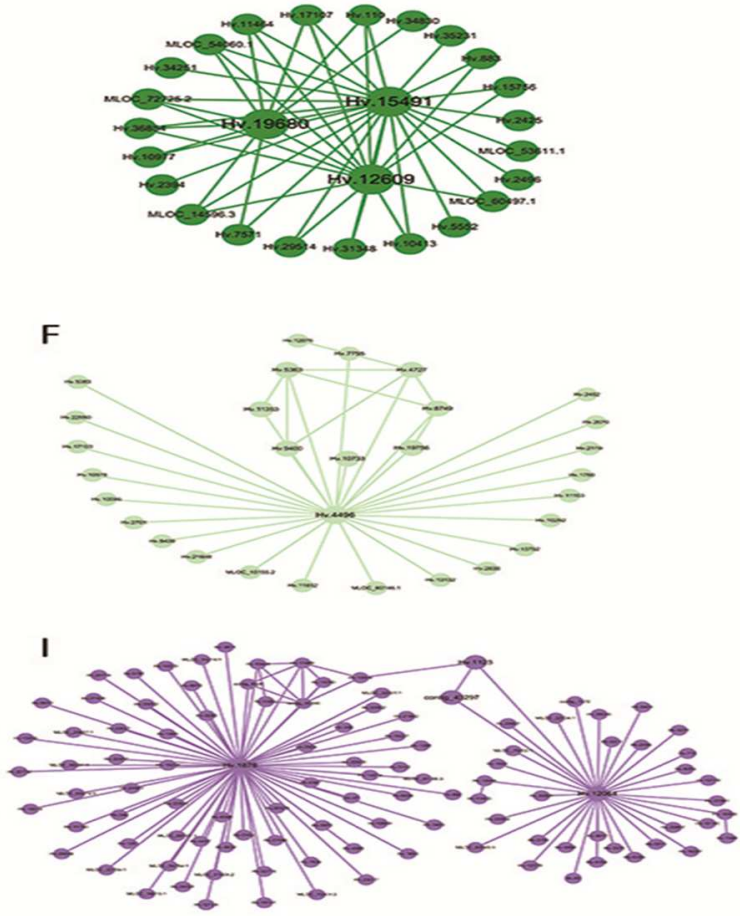

Fig. 3. Co-expression network analysis of green, light green and purple modules. (A, D, G) Heatmaps respectively represent genes that are significantly overexpressed in the three modules. (C, F, I) Eigen-gene expression profiles for the three modules at different treatments, respectively. The $y$-axis indicates the value of the module Eigen-gene and the $x$-axis indicates the samples under different treatments. $(B, E, H)$ The correlation networks corresponding to the three modules, respectively. 


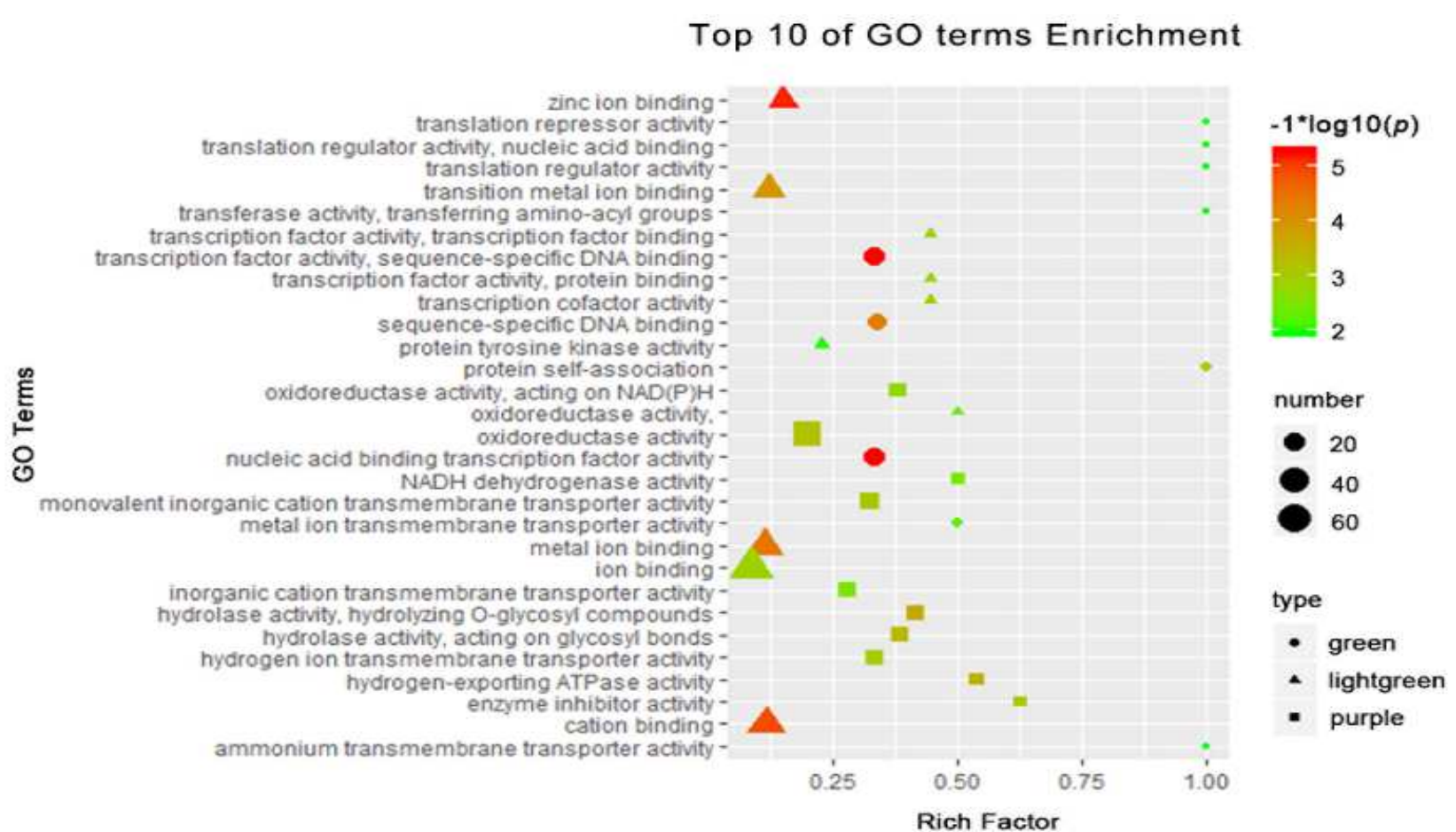

Fig. 4. Cross-comparison of pathway enrichment analysis among differentially expressed genes in barley dynamic Inflorescence development.

Supplementary Materials

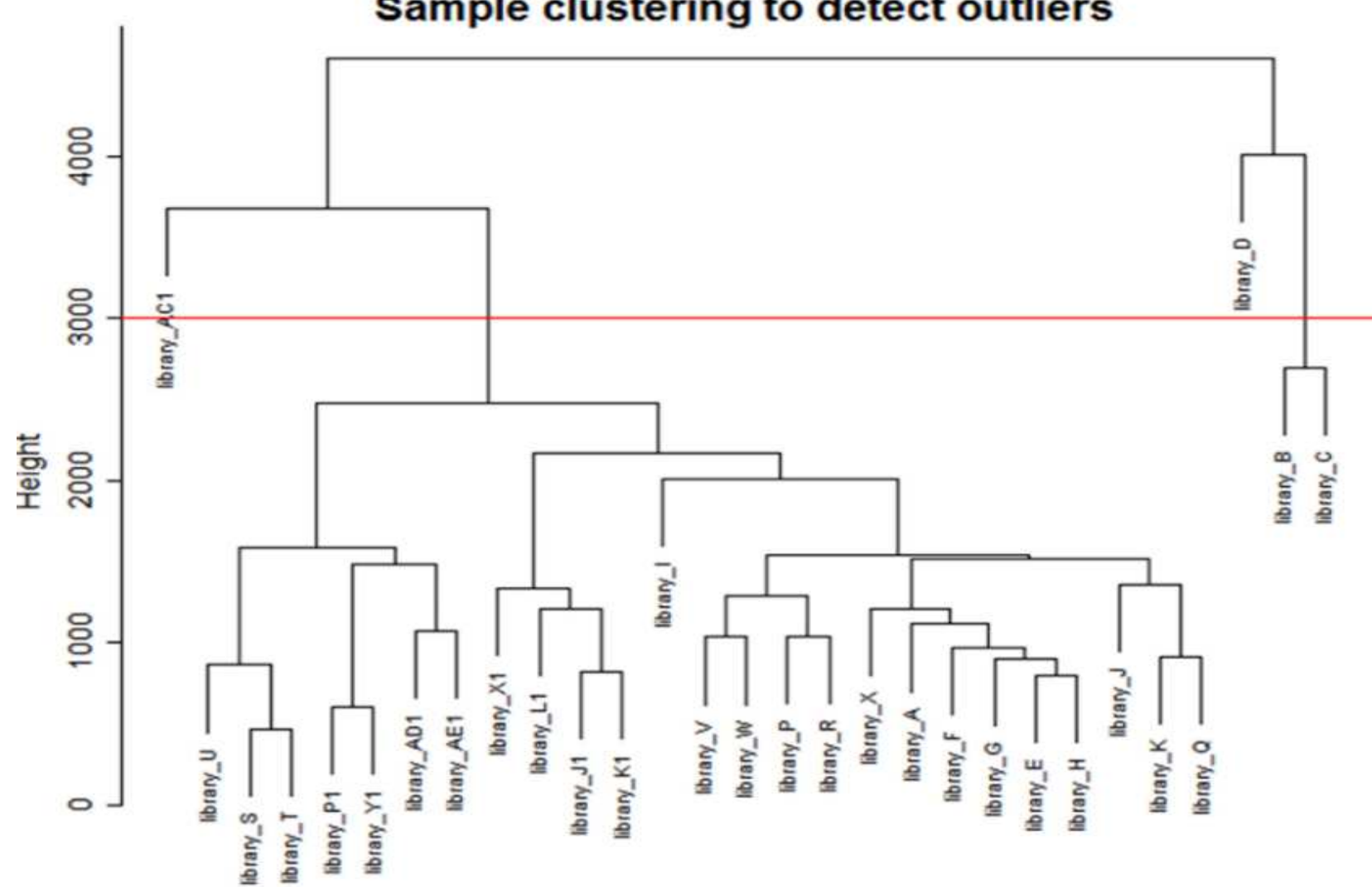

Supplemental Fig. 1. Cluster analysis of 25 samples 


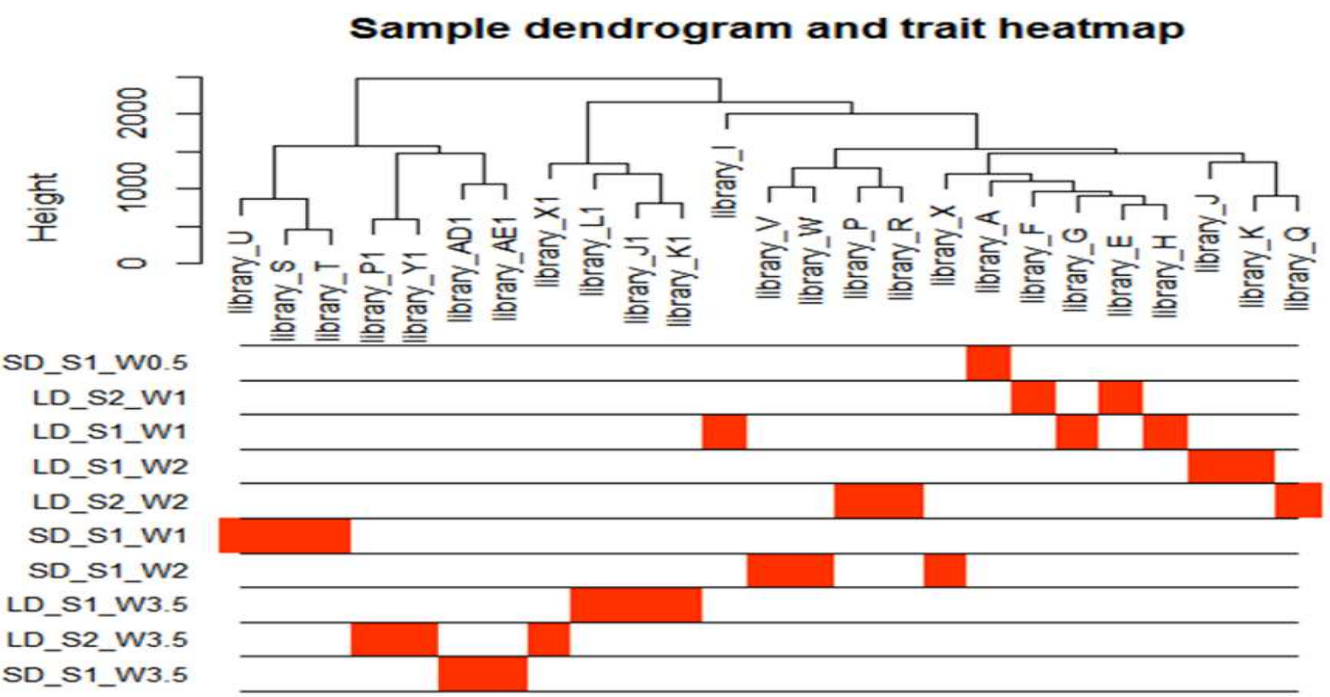

Supplemental Fig. 2. Systematic clustering tree and trait heat map of 25 samples, the samples correspond to the treatments.
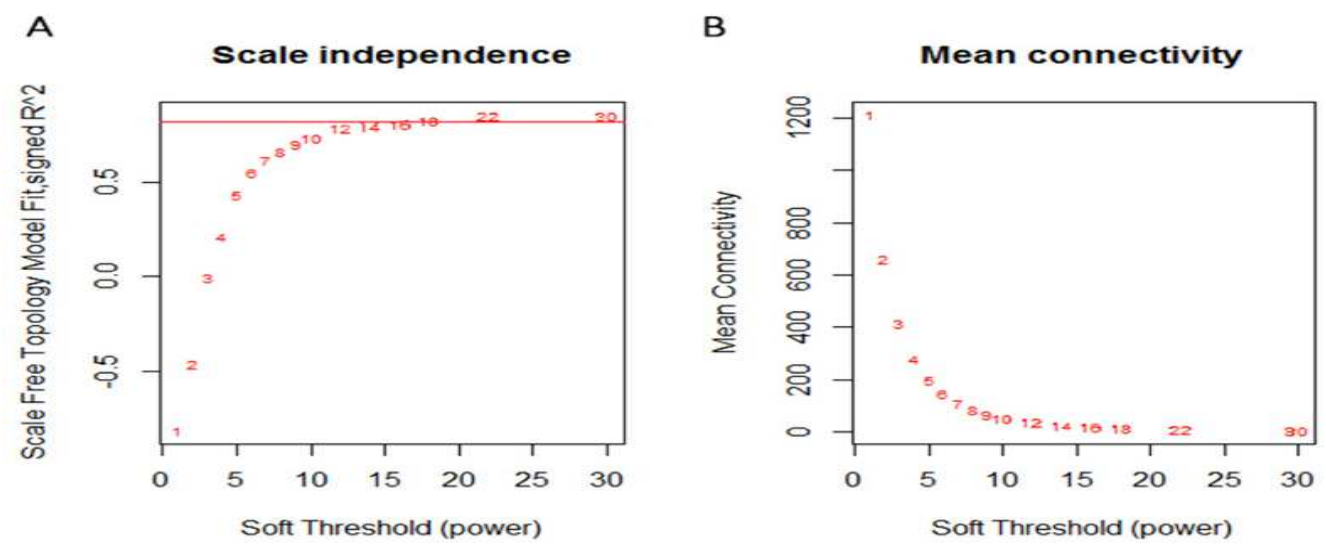

Supplemental Fig. 3. Determine the soft threshod. (A) To determine the scale independence, and the vertical axis represents the square of the correlation coefficient between $\log (i)$ and $\log (p(i))$, which should satisfy the minimum value of $>0.8$ and try to approximate the condition of $\mathbf{1}($ maximum $=1)$, (B) To determine the average connectivity, and the vertical axis represents the mean of the adjacent coefficients of all genes in the model, which reflects the average connection level of the network.

Supplementary Table 1. Detailed information sheet for 29 samples.

\begin{tabular}{llll}
\hline Stage & Photoperiod & Library & Sample \\
\hline \multirow{2}{*}{ W0.5 } & & library_A & S42-IL017_rep1 \\
& SD & library_B & S42-IL017_rep2 \\
& & library_C & S42-IL017_rep3 \\
& & library_D & Scarlett_rep1* \\
& & library_E & Scarlett_rep2 \\
W1 & library_F & Scarlett_rep3 \\
& & library_G & S42-IL017_rep1 \\
& & library_H & S42-IL017_rep2 \\
& & library_I & S42-IL017_rep3 \\
& SD & library_S & S42-IL017_rep1 \\
W2 & library_T & S42-IL017_rep2 \\
& & library_U & S42-IL017_rep3 \\
& & library_J & S42-IL017_rep1 \\
& LD & library_K & S42-IL017_rep2
\end{tabular}




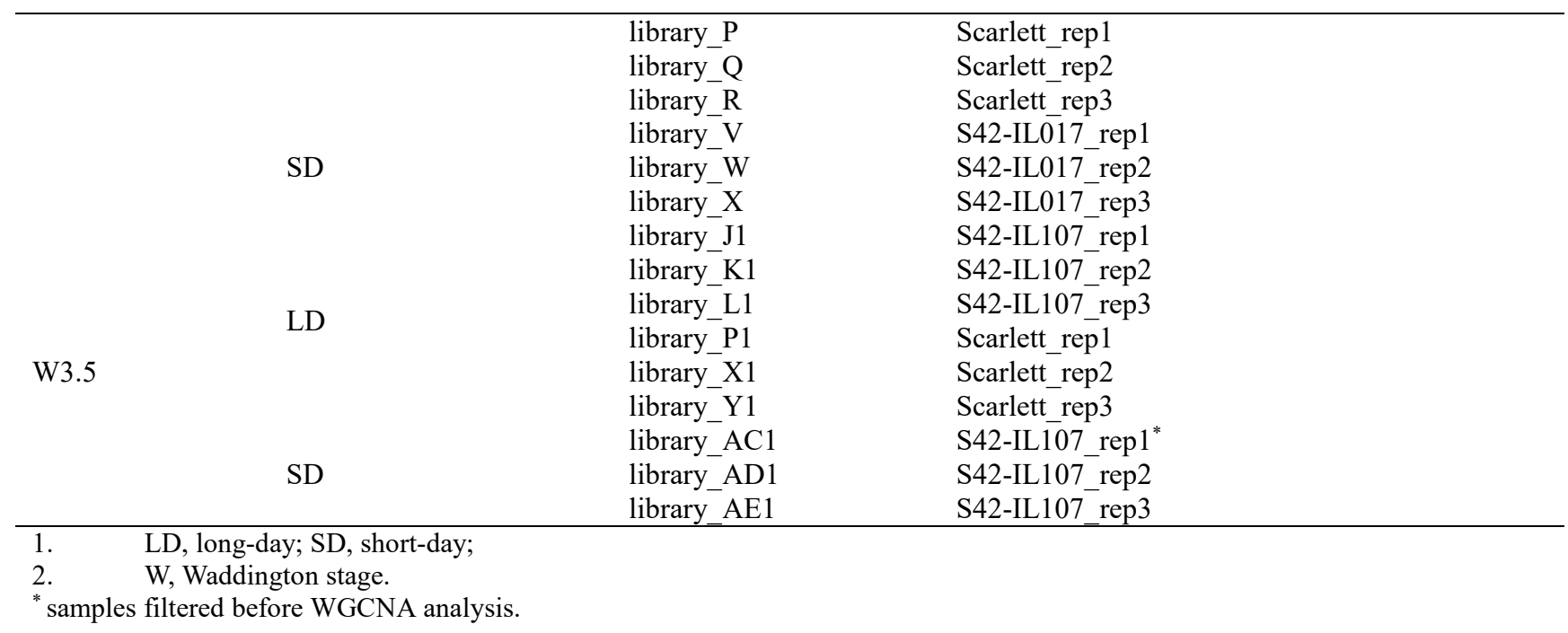

\section{DISCUSSION}

Barley is one of the oldest cereals in the world. It has wide adaptability and strong resistance to stress, and is also one of the raw materials for beer production. Inflorescence development and floral organ formation directly affect the sexual reproduction of plants, thus affecting yield. The published barley transcriptome gene expression profiles are generally used to describe the expression of genes in physiological processes and are rarely used to explore molecular mechanisms (Greenup et al., 2011). The dataset obtained by WGCNA can provide a theoretical basis for the hypotheses on the regulation of the development of barley inflorescence and could guide further experimental design required for functional verification.

WGCNA reveals molecular modules that are closely related to inflorescence development: Co-expression gene cluster analysis demonstrated the temporal distribution of core molecular events during inflorescence development in barley. When we built the matrix, we used published RNA-seq data that were more reliable than chip data and detected more number of genes ( $\mathrm{Ju}$ et al., 2018). Different stages of inflorescence development have different characteristics. The stages $\mathrm{W} 1$ to $\mathrm{W} 3.5$ are processes in which plants change from a vegetative growth stage to an early reproductive stage. MSAs act as a "marker" in which transcriptional changes can represent transcriptional changes during inflorescence development in barley. The gene expression characteristics of the variant material S42-IL107 in the W1 and W3.5 stages under LD conditions were significantly correlated with the green and purple modules, respectively. This indicates that the two modules are enriched for genes that are highly expressed in the corresponding inflorescence developmental stages.
Transcriptional regulation in different stages: Analysis of genes involved in transcriptional regulation indicates that transcription factors play an important role in flower bud formation. The expression profile during different developmental stages indicates the specificity of the transcription factor. MTRs and GO analysis identified key transcription factors within the module that are associated with the control of flowering time. We found that during the W0.5 and W1 stages, genes encoding $M A D S$-box transcription factor family protein ( $H v .110$, $H v .15491$ and $H v .19680$ in the green module) were identified as hub genes. These three MADS-box TFs are homologous to SHORT VEGETATIVE PHASE (SVP), and are highly expressed at the apex of the vegetative growth phase and gradually downregulated during MSA development. (Digel et al., 2015). MADS-box TF family protein has been shown to play an important role in floral evolution (Theissen et al., 2000). The MADS-box TF inhibitor $C O$ (SOC1), which is dependent on GAdependent pathway overexpression, affects flowering time under short daylight conditions (Zhao et al., 2014). $H v .12609$, a homolog of $V R N 1$, encodes an AP2/B3-like transcriptional factor family protein and is involved in epigenetic gene silencing in Arabidopsis (Digel et al., 2015). AP2/B3-like transcriptional factor family protein contains a B3 domain, and proteins containing this domain have been shown to be involved in early flower development (Wang et al., 2012). In the purple module, Hv.12064 encodes a MYB-like HTH transcriptional regulator family protein, which is involved in leaf growth and patterning, and auxin signaling (Digel et al., 2015). The above genes were also identified by Digel et al. (2015) and confirmed the reliability of the WGCNA analysis.

In addition, we found genes that also have a potential function during inflorescence development. GRF2 (Hv.15756) in the green module, identified as a 
hub gene, represents a class of plant-specific transcription factors that are members of the growth regulator family. GRF2 is a type of growth regulating factor encoding a transcription activator. It is unclear how GRF2 protein regulates growth in barley. However, the overexpression of $A t G R F 2$ has been shown to accelerate embryonic cotyledon development, germination of apical meristem differentiation and vegetative growth in Arabidopsis (Kim et al., 2010). Due to the specific expression of GRF2 during the W1 stage, we infer that it also has a function in the regulation of inflorescence development. Two flower-related genes were identified in the light green module of the W2 and W3.5 stages, including proline-rich family protein $(H v .4496)$ and ARM repeat superfamily protein $(H v .17103)$. The proline-rich protein is one of the structural cell wall proteins in plants (Chen et al., 1985). In rice, the proline-rich family protein has been shown to be involved in flower organ development, and after knocking out the gene, rice flower buds withered (Gothandam et al., 2010). However, its mechanism of action in barley is still unclear. The ARM protein is located in the nucleus and is broadly expressed throughout vegetative and floral tissues. In the purple module, XTH9 (Hv.12170) transcripts were identified as one of the hub genes and accumulated in the MSAs. During the breeding season, the transcript level of the shoot tips further increased, and they were also detected in flower buds, pedicels and internodes with flowers. It has been shown to be coordinated with plant development, including the growth of meristems and the elongation of the cells of the inflorescence stems. (Hyodo et al., 2003). The G2484-1 protein contains an agenet domain, but its function is unclear. However, the gene AIP1 identified in Arabidopsis thaliana contains the Agenet/Tudor domain and is expressed in reproductive tissues, with its downregulation delaying flower development (Brasil et al., 2015). Therefore, we conclude that the specific expression of the G2484-1 protein is similar to that of AIP1 and may play a regulatory role in flower development.

Acknowledgments: Thanks to the RNA-seq data of the Max Planck Institute for Plant Breeding Research. This research was financially supported by Second Tibetan Plateau Scientific Expedition and Research (STEP) program (Grant No. 2019QZKK0303), Science and Technology Service Network Initiative (STS) of CAS, the Key Research and Development and Transformation Project of Qinghai Province (Grant No. 2019-NK-117), and Provincial Natural Science Foundation of Qinghai (Grant No. 2020-ZJ-908).

\section{REFERENCES}

Borràs-Gelonch, G., M. Denti, W.T.B. Thomas and I. Romagosa (2012). Genetic control of pre- heading phases in the Steptoe $\times$ Morex barley population under different conditions of photoperiod and temperature. Euphytica. 183 (3): 303-321.

Brasil, J.N., L.M. Cabral, N.B. Eloy, L.M.F. Primo, I.L. Barroso-Neto, L.P.P. Grangeiro, N. Gonzalez, D. Inzé, P.C.G. Ferreira and A.S. Hemerly (2015). AIP1 is a novel Agenet/Tudor domain protein from Arabidopsis that interacts with regulators of DNA replication, transcription and chromatin remodeling. BMC Plant Biol. 15 (1): 270.

Bao, W., M.J. Greenwold and R.H. Sawyer (2017). Using scale and feather traits for module construction provides a functional approach to chicken epidermal development. Funct Integr Genomics. 17 (6): 641-651.

Chen, J. and J.E. Varner (1985). Isolation and characterization of cDNA clones for carrot extensin and a proline-rich $33-\mathrm{kDa}$ protein. Proc Natl Acad Sci. 82(13): 4399-4403.

Digel, B., A. Pankin, and M. Von Korff (2015). Global transcriptome profiling of developing leaf and shoot apices reveals distinct genetic and environmental control of floral transition and inflorescence development in barley. Plant Cell. 27(9): 2318-2334.

Fischer, R.A. (1985). Number of kernels in wheat crops and the influence of solar radiation and temperature. J. Agric Sci. 105(2): 447-461.

Feng, G., L. Huang, J. Li, J. Wang, L. Xu, L. Pan, X. Zhao, X. Wang, T. Huang and X. Zhang (2017). Comprehensive transcriptome analysis reveals distinct regulatory programs during vernalization and floral bud development of orchardgrass (Dactylis glomerata L.). BMC Plant Biol. 17(1): 1-19.

González, F.G., G.A. Slafer and D.J. Miralles (2002). Vernalization and photoperiod responses in wheat pre-flowering reproductive phases. Field Crops Res. 74(2): 183-195.

Gothandam, K.M., E. Nalini, S. Karthikeyan and J.S. Shin (2010). OsPRP3, a flower specific prolinerich protein of rice, determines extracellular matrix structure of floral organs and its overexpression confers cold-tolerance. Plant Mol Biol. 72(1-2): 125-135.

Greenup, A.G., S. Sasani, S.N. Oliver, S.A. Walford, A.A. Millar and B. Trevaskis (2011). Transcriptome analysis of the vernalization response in barley (Hordeum vulgare) seedlings. PLoS One. 6(3): e17900.

Ghan, R., J. Petereit, R.L. Tillett, K.A. Schlauch, D. Toubiana, A. Fait and G.R. Cramer (2017). The common transcriptional subnetworks of the grape berry skin in the late stages of ripening. BMC Plant Biol. 17(1): 94. 
Giulietti, M., G. Occhipinti, G. Principato and F. Piva (2017). Identification of candidate miRNA biomarkers for pancreatic ductal adenocarcinoma by weighted gene coexpression network analysis. Cell Oncol. 40(2): 181-192.

Hyodo, H., S. Yamakawa, Y. Takeda, M. Tsuduki, A. Yokota, K. Nishitani and T. Kohchi (2003). Active gene expression of a xyloglucan endotransglucosylase/hydrolase gene, XTH9, in inflorescence apices is related to cell elongation in Arabidopsis thaliana. Plant Mol Biol. 52(2): 473-482.

Ju, Z., D. Cao, Y. Liang, H. Tian, B. Zhu and Y. Luo (2018). The transcription factors related to tomato fruit ripening were analyzed by WGCNA. Chinese Institute of Food Science and Technology. 18(6): 240-248.

Kim, J.H., D. Choi and H. Kende (2010). The AtGRF family of putative transcription factors is involved in leaf and cotyledon growth in Arabidopsis. Plant J. 36(1): 94-104.

Karsai, I., P.M. Hayes, J. Kling, I.A. Matus, K. Mészáros, L. Láng, Z. Bedő and K. Sato (2004), Genetic variation in component traits of heading date in subsp. accessions Characterized in Controlled Environments. Crop Sci. 44(5): 1622-1632.

Kitagawa, S., S. Shimada and K. Murai (2012). Effect of Ppd-1 on the expression of flowering-time genes in vegetative and reproductive growth stages of wheat. Genes Genet Syst. 87(3): 161-168.

Laurie, D.A., N. Pratchett, J.W. Snape and J.H. Bezant (1995). RFLP mapping of five major genes and eight quantitative trait loci controlling flowering time in a winter $\mathrm{x}$ spring barley (Hordeum vulgare L.) cross. Genome. 38(3): 575-585.

Langfelder, P. and S. Horvath (2008). WGCNA: an R package for weighted correlation network analysis. BMC Bioinformatics. 9(1): 559.

Lin, X., J. Li, Q. Zhao, J.R. Feng, Q. Gao and J.Y. Nie (2018). WGCNA reveals key roles of IL8 and MMP-9 in progression of involvement Area in colon of patients with ulcerative colitis. Curr Med Sci. 38: 252-258.

Ping, Y., Y. Deng, L. Wang, H. Zhang, Y. Zhang, C. Xu and X. Li (2015). Identifying core gene modules in glioblastoma based on multilayer factormediated dysfunctional regulatory networks through integrating multi-dimensional genomic data. Nucleic Acids Res. 43(4): 1997-2007.

Sebastián, A., and D.J. Miralles (2008). Critical period for grain number establishment of near isogenic lines of two- and six-rowed barley. Field Crops Res. 107(3): 196-202.
Seki, M., M. Chono, T. Nishimura, M. Sato, Y. Yoshimura, H. Matsunaka, M. Fujita, S. Oda, K. Kudo, C. Kiribuchi-Otobe, H. Kojima, H. Nishida and K. Kato (2013). Distribution of photoperiod-insensitive allele Ppd-Ala and its effect on heading time in Japanese wheat cultivars. Breed Sci. 63(3): 309-316.

Slafer, G.A. and H.M. Rawson (1994). Sensitivity of wheat phasic development to major environmental factors: a re-examination of some assumptions made by physiologists and modellers. Funct. Plant Biol. 21(4): 393-426.

Theissen, G., A. Becker, A.D. Rosa, A. Kanno, J.T. Kim, T. Münster and H. Saedler (2000). A short history of MADS-box genes in plants. Plant Mol Biol. 42(1): 115-149.

Turner, A., J. Beales, S. Faure, R.P. Dunford and D.A. Laurie (2005). The pseudo-response regulator Ppd-H1 provides adaptation to photoperiod in barley. Science. 310(5750): 1031-1034.

Tang, Y., X. Zeng, Y. Wang, L. Bai, Q. Xu, Z. Wei and T. Nyima (2017). Transcriptomics analysis of hulless barley during grain development with a focus on starch biosynthesis. Funct Integr Genomics. 17(1): 107-117.

Wang, Y., D. Deng, R. Zhang, S. Wang, Y. Bian and Z. Yin (2012). Systematic analysis of plant-specific B3 domain-containing proteins based on the genome resources of 11 sequenced species. Mol Biol Rep. 39(5): 6267-6282.

Wang, J., X. Zhang, M. Shi, L. Gao, X. Niu, R. Te, L. Chen and W. Zhang (2014). Metabolomic analysis of the salt-sensitive mutants reveals changes in amino acid and fatty acid composition important to long-term salt stress in Synechocystis sp. PCC 6803. Funct Integr Genomics. 14(2): 431-440.

Yuan, H., X. Zeng, Q. Yang, Q. Xu, Y. Wang, D. Jabu, Z.Sang and N. Tashi (2018). Gene coexpression network analysis combined with metabonomics reveals the resistance responses to powdery mildew in Tibetan hulless barley. Sci Rep. 8(1): 14928.

Zhao, S., Y. Luo, Z. Zhang, M. Xu, W. Wang, Y. Zhao, L. Zhang, Y. Fan and L. Wang (2014). ZmSOC1, a MADS-box transcription factor from Zea mays, promotes flowering in Arabidopsis. Int $\mathrm{J}$ Mol Sci. 15(11): 19987-20003.

Zhang, J., W. Zhao, R. Fu, C. Fu, L. Wang, H. Liu, S. Li, Q. Deng, S. Wang, J. Zhu, Y. Liang, P. Li and A. Zheng (2018). Comparison of gene conetworks reveals the molecular mechanisms of the rice (Oryza sativa L.) response to Rhizoctonia solani AG1 IA infection. Funct Integr Genomics. 18(5): 545-557. 